\title{
Polarization-Independent Multiwavelength-Switchable Filter Based on Polarization Beam Splitter and Fiber Coupler
}

\begin{abstract}
Yong Wook Lee*
Abstract - A polarization-independent multiwavelength-switchable fiber filter is proposed based on a polarization beam splitter and fiber coupler, which can function as a polarization-independent transmission or reflection-type interleaving filter. The proposed filter consists of a polarization beam splitter and a Sagnac birefringence loop composed of a 50:50 coupler, high birefringent fibers, and two quarter-wave plates. In the proposed filter, a transmission-type interleaver with a channel isolation $>18 \mathrm{~dB}$ or a reflection-type one with a channel isolation of $\sim 3 \mathrm{~dB}$, whose channel spacing and switching displacement were 0.8 and $0.4 \mathrm{~nm}$ in common, respectively, could be obtained. A channel interleaving operation could be performed by the proper control of waveplates within the Sagnac birefringence loop.
\end{abstract}

Keywords: multiwavelength, switching, optical filter, polarization beam splitter, high birefringent fiber

\section{Introduction}

Multiwavelength-switchable fiber filters are of great importance for the next-generation of wavelength-routed wavelength-division-multiplexed networks as their dynamic transmission property can provide high system performance. Recently, fiber birefringent filters based on a Sagnac birefringence loop (SBL) structure have gained attention for their simple design and ease of use as multiwavelength-selective filters. Initially considered as a switching device, the theory and application of Sagnac fiber loop reflectors, fabricated by forming a fiber loop between the output ports of a directional coupler, have been well documented and understood [1], with many useful applications found in the area of photonics. In the meantime, a multiwavelength-periodic filtering function could be produced by inserting a high birefringent fiber (HBF) in the Sagnac fiber loop. Techniques for building optical filters using an SBL structure, i.e., Sagnac birefringence filters (SBFs), have been proposed and experimentally demonstrated in various forms [2][3]. However, absolute wavelength control of the periodic reflection or transmission band in these SBFs requires some mechanical or thermal techniques [4]. Recently, a multiwavelength-switchable filter controlled by the adjustment of the light polarization circulating the fibers comprising the filter, and thus causing no physical harm to the durability of the HBF, was reported by incorporating a polarization beam splitter (PBS) and SBF to implement the multiwavelength-switchable fiber ring laser [5]. In the previous study [5], however, a filter operating only in a reflection mode was used and analyzed, and uniform spectral characteristics could not be obtained in a broadband $>10 \mathrm{~nm}$ due to the wavelength dependency of

* School of Electrical Engineering, Pukyong National University, San 100 Yongdang-dong, Nam-gu, Busan 608-739, Korea.

(yongwook@pknu.ac.kr)

Received 7 April 2009; Accepted 8 July 2009 the waveplates used in the experiments. In this paper, a polarization-independent multiwavelength-switchable filter was demonstrated, which could operate in both transmission and reflection modes and show uniform spectral outputs in a broadband $>20 \mathrm{~nm}$ by using high performance waveplates nearly achromatic over $20 \mathrm{~nm}$ around $1550 \mathrm{~nm}$. The proposed filter consists of a PBS and an SBL composed of a 50:50 coupler, HBFs, and two quarter-wave plates (QWPs). With proper adjustment of the polarization controllers (PCs) composed of two QWPs within the SBL, the channel wavelength can be switched so that the switching displacement should be half of the spectral period. This half-period switching that makes the channel interleave is known as interleaving. In the proposed filter, a transmission-type interleaving filter (interleaver) with a channel isolation $>18 \mathrm{~dB}$ or a reflection-type one with a channel isolation of $\sim 3 \mathrm{~dB}$, whose channel spacing and switching displacement were 0.8 and $0.4 \mathrm{~nm}$ in common, respectively, could be obtained in a wavelength range of $20 \mathrm{~nm}$.

\section{Principles of Operation}

\subsection{Mathematical Formulation of Filter Responses}

As shown in a schematic diagram of the proposed filter in Fig. 1, the filter consists of a PBS and an SBL composed of a 50:50 coupler, HBFs, and two QWPs. The PBS is a compact optical device that can split the input light into two orthogonal polarization components or combine them into one output fiber. The SBL in the dotted box in Fig. 1 acts as a polarization-independent multiwavelength-periodic filter [2]. As can be seen in Fig. 1, there are 8 paths of the propagating light when the light is introduced into the input port of the filter (8 paths: 1-3-4-1, 1-3-4-2, 1-4-3-1, 1-4-3-2, 2-3-4-1, 2-3-4-2, 2-4-3-1, and 2-4-3-2). Only the reflected or transmitted light should be included in the theoretical calculation of reflectivity or transmittivity with the proper 
consideration on polarization, respectively. If optical components comprising the filter are assumed as ideal, i.e., lossless, including a 50:50 coupler, QWPs, and a PBS, and the above 8 paths are considered in turn, the Jones matrices $R E$ and $T R$ for reflection and transmission, respectively, can be represented as follows according to Jones matrix formulation [6]:

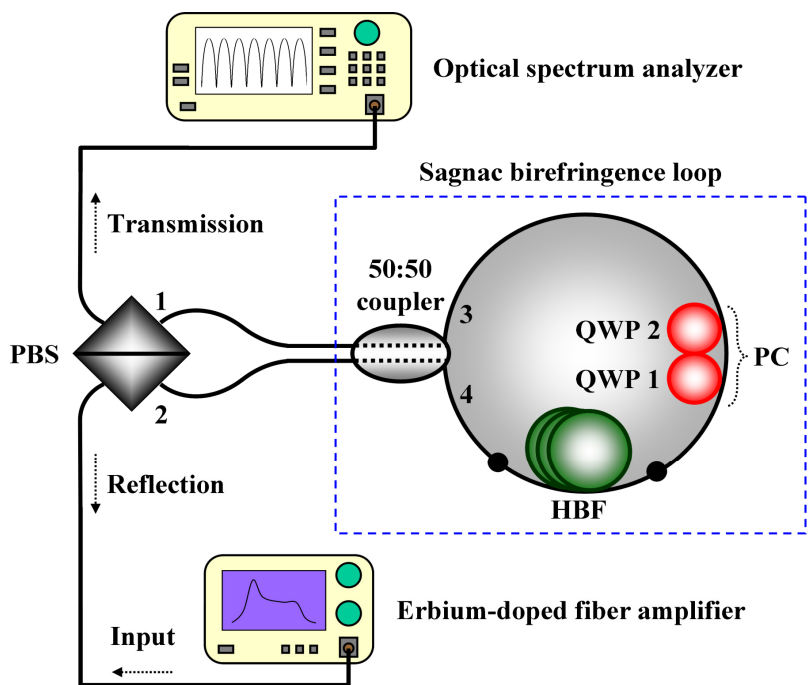

Fig. 1. Schematic diagram of the proposed multiwavelengthswitchable filter (dots: splicing points between HBFs and single-mode fibers)

$$
\begin{gathered}
R E=\frac{i}{2} T_{H} T_{H B F}\left(\theta_{h}\right) T_{Q 1}\left(\theta_{1}\right) T_{Q 2}\left(\theta_{2}\right) T_{H} \\
+\frac{1}{2} T_{V} T_{H B F}\left(\theta_{h}\right) T_{Q 1}\left(\theta_{1}\right) T_{Q 2}\left(\theta_{2}\right) T_{H}+\frac{i}{2} T_{H} T_{Q 2}\left(-\theta_{2}\right) T_{Q 1}\left(-\theta_{1}\right) T_{H B F}\left(-\theta_{h}\right) T_{H} \\
+\frac{i^{2}}{2} T_{V} T_{Q 2}\left(-\theta_{2}\right) T_{Q 1}\left(-\theta_{1}\right) T_{H B F}\left(-\theta_{h}\right) T_{H}+\frac{i^{2}}{2} T_{H} T_{H B F}\left(\theta_{h}\right) T_{Q 1}\left(\theta_{1}\right) T_{Q 2}\left(\theta_{2}\right) T_{V} \\
+\frac{i}{2} T_{V} T_{H B F}\left(\theta_{h}\right) T_{Q 1}\left(\theta_{1}\right) T_{Q 2}\left(\theta_{2}\right) T_{V}+\frac{1}{2} T_{H} T_{Q 2}\left(-\theta_{2}\right) T_{Q 1}\left(-\theta_{1}\right) T_{H B F}\left(-\theta_{h}\right) T_{V} \\
+\frac{i}{2} T_{V} T_{Q 2}\left(-\theta_{2}\right) T_{Q 1}\left(-\theta_{1}\right) T_{H B F}\left(-\theta_{h}\right) T_{V} \\
+\frac{1}{2} T_{H} T_{H B F}\left(\theta_{h}\right) T_{Q 1}\left(\theta_{1}\right) T_{Q 2}\left(\theta_{2}\right) T_{H}+\frac{i}{2} T_{V} T_{Q 2}\left(-\theta_{2}\right) T_{Q 1}\left(-\theta_{1}\right) T_{H B F}\left(-\theta_{h}\right) T_{H}\left(\theta_{h}\right) T_{Q 1}\left(\theta_{1}\right) T_{Q 2}\left(\theta_{2}\right) T_{H} \\
+\frac{i^{2}}{2} T_{H} T_{Q 2}\left(-\theta_{2}\right) T_{Q 1}\left(-\theta_{1}\right) T_{H B F}\left(-\theta_{h}\right) T_{H}+\frac{i^{2}}{2} T_{V} T_{H B F}\left(\theta_{h}\right) T_{Q 1}\left(\theta_{1}\right) T_{Q 2}\left(\theta_{2}\right) T_{V} \\
+\frac{i}{2} T_{H} T_{H B F}\left(\theta_{h}\right) T_{Q 1}\left(\theta_{1}\right) T_{Q 2}\left(\theta_{2}\right) T_{V}+\frac{1}{2} T_{V} T_{Q 2}\left(-\theta_{2}\right) T_{Q 1}\left(-\theta_{1}\right) T_{H B F}\left(-\theta_{h}\right) T_{V} \\
+\frac{i}{2} T_{H} T_{Q 2}\left(-\theta_{2}\right) T_{Q 1}\left(-\theta_{1}\right) T_{H B F}\left(-\theta_{h}\right) T_{V}
\end{gathered}
$$

where $T_{H}, T_{V}, T_{H B F}, T_{Q 1}$, and $T_{Q 2}$ are Jones matrices of the horizontal polarizer, vertical polarizer, HBF, QWP1, and QWP2, respectively. And $\theta_{h}, \theta_{1}$, and $\theta_{2}$ are, respectively, fast-axis orientation (azimuthal) angles of the HBF, QWP1, and QWP2 with respect to the horizontal axis of the PBS, assumed as the reference axis. Here, $T_{H B F}\left(\theta_{h}\right)=R\left(-\theta_{h}\right)$ $\cdot\left[\begin{array}{cc}e^{i \Gamma / 2} & 0 \\ 0 & e^{-I / 2}\end{array}\right] R\left(\theta_{h}\right)$ and $R(\theta)=\left[\begin{array}{cc}\cos \theta & \sin \theta \\ -\sin \theta & \cos \theta\end{array}\right] . \Gamma(=2 \pi B L / \lambda)$ is the phase difference between the fast and slow-axis components of the light at the free space wavelength $\lambda$, which propagates through the HBF with the birefringence $B$ and length $L$.

Using $R E$ and $T R$, the reflectivity $R$ and transmittivity $T$ of the filter can be calculated as follows:

$$
\begin{gathered}
R=1-\left[\sin \left(\theta_{1}-\theta_{2}\right) \cos \left(\theta_{1}-\theta_{2}\right) \cos \left(\frac{\Gamma}{2}\right)+\sin \left(2 \theta_{h}-\theta_{1}-\theta_{2}\right) \cos \left(\theta_{1}-\theta_{2}\right) \sin \left(\frac{\Gamma}{2}\right)\right]^{2} \\
T=\left[\sin \left(\theta_{1}-\theta_{2}\right) \cos \left(\theta_{1}-\theta_{2}\right) \cos \left(\frac{\Gamma}{2}\right)+\sin \left(2 \theta_{h}-\theta_{1}-\theta_{2}\right) \cos \left(\theta_{1}-\theta_{2}\right) \sin \left(\frac{\Gamma}{2}\right)\right]^{2}
\end{gathered}
$$

Depending on the set of $\theta_{1}$ and $\theta_{2}$, both amplitude (modulation depth) and maxima/minima transmission wavelengths of the interference pattern in the filter spectrum change. In Eqs. (3) and (4), it is notable that while intensity distribution of the light going from two output ports of the PBS and into two ports of the SBL depends on input polarization, the proposed filter is independent of input polarization, regardless of operation type (reflection or transmission-type).

\subsection{Types of Filter Operation}

On the basis of Eq. (3), the function of the reflectiontype filter, as a rule, can be classified as four categories, depending on the combination of the QWP angles. The four categories are as follows: [a] a reflection-type interleaving filter, [b] a reflection-type comb filter with large channel isolation $(>30 \mathrm{~dB}),[\mathrm{c}]$ a $100 \%$ reflector, and [d] a reflection-type comb filter pair which can make the channel isolation of each one the same but cannot make each one interleave with the other. For category [a], two interleaved interference spectra like $\pi$ phase-shifted spectrum pair can be achieved using suitably chosen two sets of $\theta_{1}$ and $\theta_{2}$, designated as interleaving sets, and this type of operation cannot be achieved using only the SBF. Among the four categories, category [a] is used for implementing an interleaving filter.

Fig. 2(a) shows the calculated reflection spectra of the filter at two interleaving sets of $\left(\theta_{1}, \theta_{2}\right)$ for interleaving operation:

$$
\begin{aligned}
& \text { solid line }\left(\theta_{h}-\left\{(n-m+1)-\frac{1}{4}\left[(-1)^{n}-(-1)^{m}\right]\right\} \frac{\pi}{2}\right. \\
&\left., \theta_{h}-\left\{(n+m-1)-\frac{1}{4}\left[(-1)^{n}+(-1)^{m}\right]\right\} \frac{\pi}{2}\right)
\end{aligned}
$$

and

$$
\begin{array}{r}
\text { dotted line }\left(\theta_{h}-\left\{(n-m)-\frac{1}{4}\left[(-1)^{n}-(-1)^{m}\right]\right\} \frac{\pi}{2}\right. \\
\left., \theta_{h}-\left\{(n+m)-\frac{1}{4}\left[(-1)^{n}+(-1)^{m}\right]\right\} \frac{\pi}{2}\right)
\end{array}
$$

where $n$ and $m$ are integers. For general combinations except interleaving sets, the filter has spectral characteristics like those shown in Fig. 2(b) (solid line: category [b], dash-dotted flat line: category [c], and two dashed and dotted lines: category [d]). The solid line is obtained at the combinations of 
QWP angles $\left(\theta_{1}, \theta_{2}\right)$ such as $\left[\theta_{h}-\left(\frac{2 n-1}{4}\right) \pi, \theta_{h}-\left(\frac{2 n-1}{4}\right) \pi\right]$, where $n$ is integer. While the channel isolation of the filter is large $(>30 \mathrm{~dB})$ in these combinations, there is no combination of QWP angles making the reflection spectrum have the same channel isolation and interleaved channel location, compared with those of the spectrum plotted in a solid line. The dash-dotted line is obtained at the combinations such as $\left[\theta_{h}-\left(\frac{n}{2}\right) \pi, \theta_{h}-\left(\frac{n}{2}\right) \pi\right]$ or $\left(\theta_{1}, \theta_{2}\right)$ satisfying $\theta_{1}-\theta_{2}=\left(\frac{2 m-1}{2}\right) \pi$ where $n$ and $m$ are integers. In these sets, the filter acts as a mirror with $100 \%$ reflectivity. Both dashed and dotted lines are obtained at the combinations of $\left(\theta_{h}-\frac{\pi}{4}, \theta_{h}-\frac{\pi}{12}\right)$ and $\left(\theta_{h}-\frac{\pi}{4}, \theta_{h}-\frac{5 \pi}{12}\right)$, respectively. At this pair of QWP combinations, the same channel isolation can be achieved at two different channel locations, but there is no combination pair making the separation between two adjacent channels a half-period, i.e., making the channels interleave. At other combinations except those described in categories [a], [b], and [c], the filter has spectral characteristics similar to those of category [d]. All cases, including categories [b], [c], and [d], were also confirmed by the experiments.

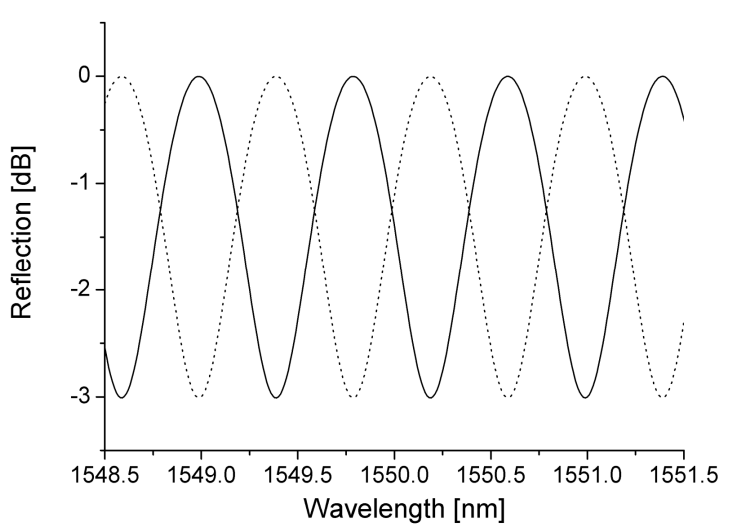

(a)

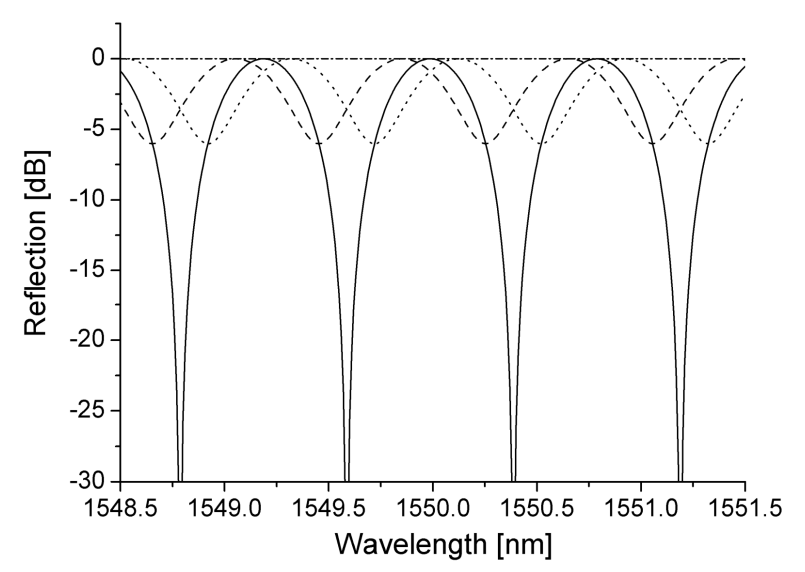

(b)

Fig. 2. Calculated reflection spectra of the proposed filter at (a) interleaving sets and (b) some other non-interleaving sets
From the comparison between Eqs. (3) and (4), it is clear that there is a complementary relationship between the sinusoidal transmission and reflection spectra. Based on Eq. (4), therefore, the function of the transmission-type filter can also be classified as four categories, depending on the combination of the QWP angles. Considering energy conservation, it is readily accepted that a transmission spectrum in each category has a complementary relationship with a reflection one in the corresponding category.

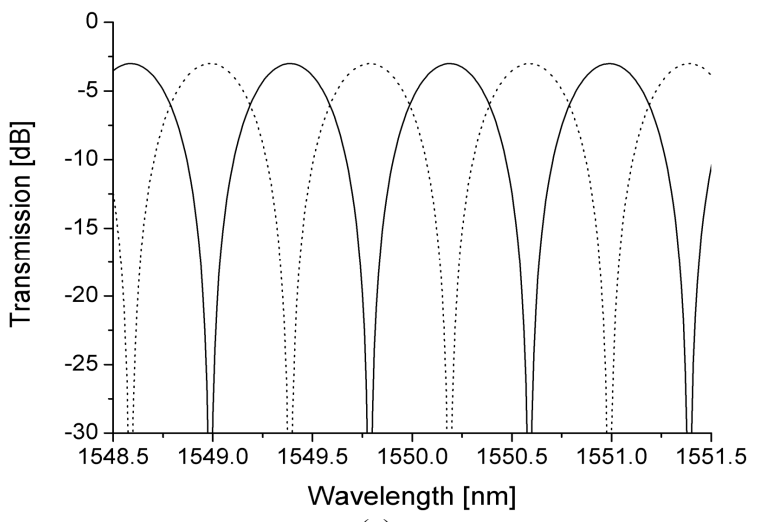

(a)

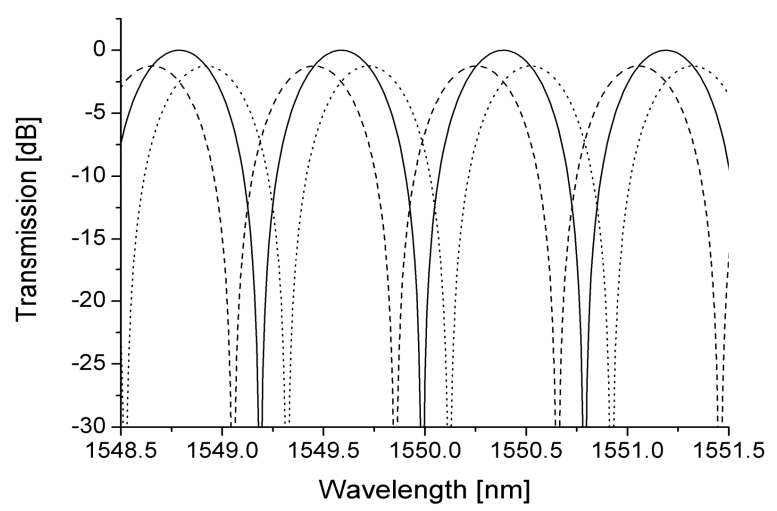

(b)

Fig. 3. Calculated transmission spectra of the proposed filter at (a) interleaving sets and (b) some other noninterleaving sets

The four categories are as follows: [a] a transmissiontype interleaving filter, [b] a transmission-type comb filter with large channel isolation (>30 dB), [c] a 100\% absorber, and [d] a transmission-type comb filter pair which can make the channel isolation of each one the same but cannot make each one interleave with the other. Similarly, with the case of the reflection-type filter, category [a] is utilized for implementing an interleaving filter among the four categories. Fig. 3(a) shows the calculated transmission spectra of the filter at two interleaving sets of $\left(\theta_{1}, \theta_{2}\right)$ for interleaving operation. Fig. 3(b) shows the calculated transmission spectra of the filter at some other non-interleaving sets (solid line: category [b], dash-dotted flat line: category [c], for simplicity, is omitted in the figure, and two dashed and dotted lines: category [d]). In all cases, QWP combinations are the same as those in the example of the reflection-type filter, and the description of the filter function is also the 
same except for category [c] in which the filter can act as a $100 \%$ absorber. Similarly, all cases were experimentally confirmed.

\section{Experiments and Discussions}

In order to demonstrate the predicted filter responses, the proposed filter was fabricated as shown in Fig. 1, by using a PBS (OZ Optics), HBFs (Fibercore), a 50:50 coupler, and two QWPs (OZ Optics). Dots in the figure indicate splicing points between the HBFs and single-mode fibers (SMFs). $B$ and $L$ of Bow-tie type HBFs used in the experiments were $\sim 4.8 \times 10^{-4}$ and $6.25 \mathrm{~m}$, respectively, and $L$ was determined so that the wavelength spacing (channel spacing) between fringe maxima becomes $0.8 \mathrm{~nm}$. The channel spacing is inversely proportional to $B$ and $L$ of the HBF and is given as $\lambda_{c} / B L$ ( $\lambda_{c}$ : center wavelength). An erbium-doped fiber amplifier was used as a broadband light source for the characterization of the filter spectra, and the transmission and reflection spectra of the filter were measured using an optical spectrum analyzer. To minimize the effect of temperature-dependent birefringence of the HBF, the ambient temperature of the HBF was constantly maintained by placing it within a thermally-insulated chamber made of styrene foam filled with water and keeping the water at a fixed temperature.

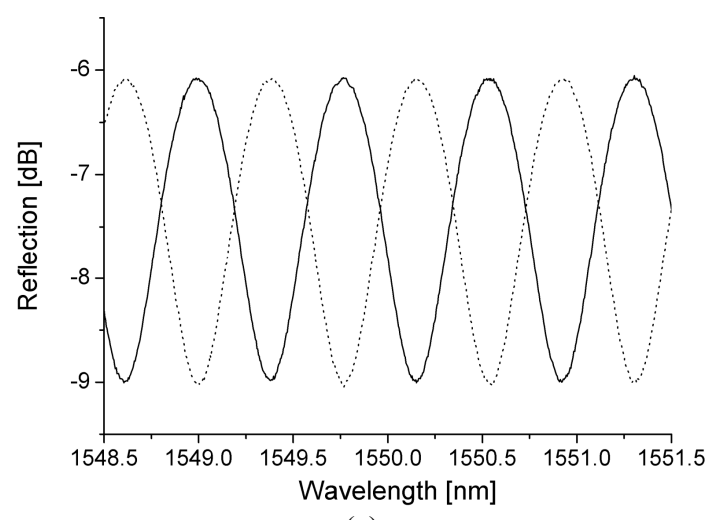

(a)

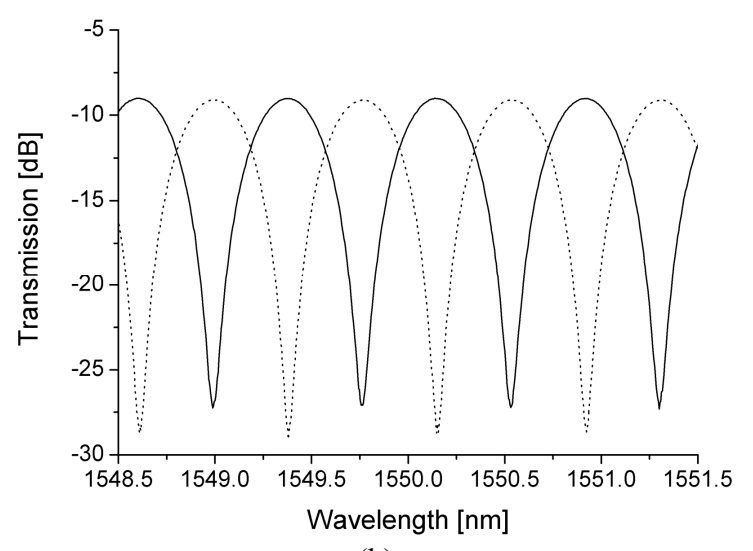

(b)

Fig. 4. Measured (a) reflection and (b) transmission spectra of the proposed filter at interleaving sets
Figs. 4(a) and 4(b) show the measured reflection and transmission spectra of the filter at two interleaving sets, respectively. QWP combinations for interleaving sets are the same in two cases (reflection and transmission) because the reflection and transmission are complementarily related. Slight differences from the theoretical results in the amplitude and wavelength location stem from the slight unevenness in insertion losses of the 50:50 coupler and the PBS and $\theta_{h}$ 's different from those used in the calculation, respectively. The typical channel isolations of the reflection and transmission-type interleavers were measured as $\sim 3$ and $\sim 18 \mathrm{~dB}$, respectively. Insertion losses of the reflection and transmission-type interleavers were measured as < $\sim 6.1$ and $\sim 9.0 \mathrm{~dB}$, respectively. In the case of the transmission-type interleaver, the channel isolation is much larger than that of the reflection-type one, but a $3-\mathrm{dB}$ insertion loss is unavoidable due to the complementary nature of the filter output. The insertion loss of the filter mainly comes from those of two QWPs (totally $\sim 1.5 \mathrm{~dB})$, PBS ( $\sim 0.93 \mathrm{~dB}$ for one way, totally $\sim 1.86 \mathrm{~dB})$, coupler $(\sim 0.58 \mathrm{~dB}$ for one way, totally $\sim 1.16 \mathrm{~dB})$, and fiber fusion splicing $(\sim 1.47 \mathrm{~dB})$ between the HBF and SMF. The insertion loss can be reduced by using low-loss waveplates and the PBS and by improving the fusion splicing of fiber splices between the HBF and SMF.

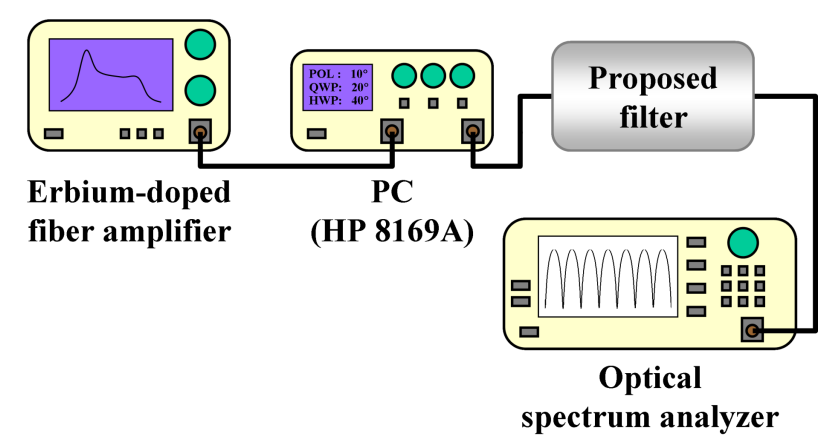

(a)

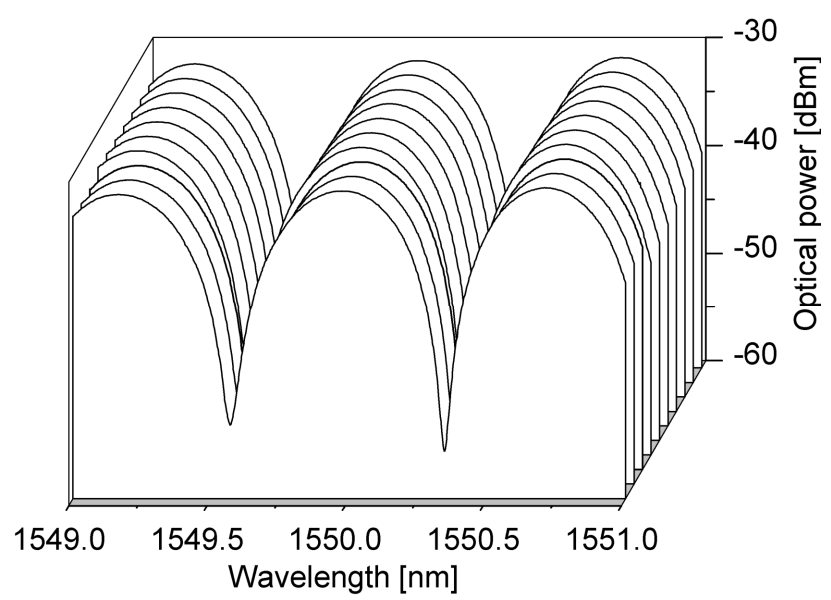

(b)

Fig. 5. (a) Experimental setup to examine the input polarization independence of the filter and (b) measured transmitted intensity spectra with respect to various input polarizations 
Especially, in order to examine the input polarization independence of the filter, the polarization sensitivity of the output intensity spectrum was measured by placing an additional PC (HP 8169 A) in front of the lead-in fiber (input port of the filter), as shown in Fig. 5(a). During the measurement, both one QWP and one half-wave plate, which were contained (with one input polarizer) within the additional PC, were rotated in a random way each time, ensuring that signal polarization had been varied over the entire Poincaré sphere. The maximum polarization sensitivity was measured as $<\sim 0.5 \mathrm{~dB}$ from the measurement results shown in Fig. 5(b), which could be affected by the polarization sensitivity of the photodetector and the imperfection of the PBS used in the experiments.

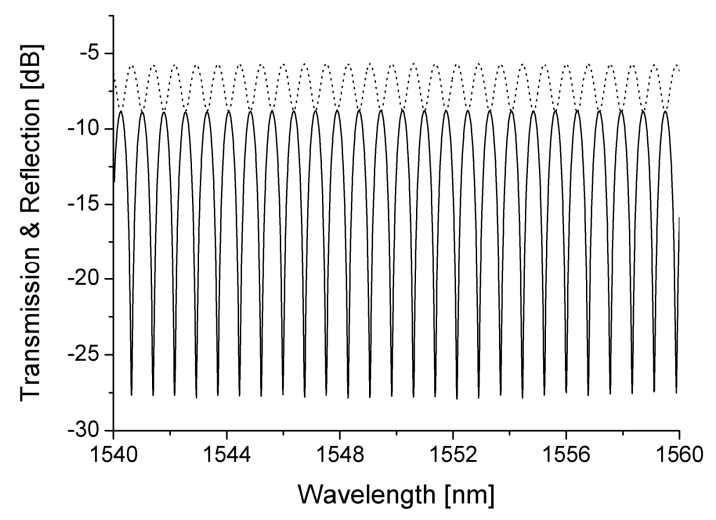

Fig. 6. Measured reflection and transmission spectra of the proposed filter at one of interleaving sets over a broad wavelength range of $20 \mathrm{~nm}$.

Fig. 6 shows the measured reflection and transmission spectra as a dotted and solid-line at one of the interleaving sets over a broad wavelength range of $20 \mathrm{~nm}$, respectively. As can be seen from the figure, it is confirmed that the reflection and transmission spectra have a complementary relationship with each other in order to keep the law of energy conservation. Spectral flatness was measured as $<$ $0.12 \mathrm{~dB}$ with two QWPs fixed. In addition, the switching speed of the proposed filter can be greatly enhanced by employing electrically controllable PCs such as those using lithium niobate structures with ns response times [7].

\section{Conclusion}

In conclusion, a broadband polarization-independent multiwavelength-periodic filter was embodied and analyzed, which could make its channel interleave over a 20 $\mathrm{nm}$ band by adjusting the QWPs within the filter. The typical channel isolations of the reflection and transmissiontype interleavers were measured as $\sim 3$ and $\sim 18 \mathrm{~dB}$, respectively. Insertion losses of the reflection and transmissiontype interleavers were measured as $<\sim 6.1$ and $\sim 9.0 \mathrm{~dB}$, respectively. Switching the capability of the channel wavelength of the proposed filter can give an expanded versatility to conventional SBFs whose spectral location is fixed. Of particular note, the proposed filters can be applied to fiber lasers and sensors as wavelength-selective interleavers [8].

\section{Acknowledgements}

This work was supported by a Korea Research Foundation Grant funded by the Korean Government (MOEHRD) (KRF-2008-331-D00376).

\section{References}

[1] D. B. Mortimore, "Fiber loop reflectors," J. Lightwave Technol., vol. 6, pp. 1217-1224, Jul. 1988.

[2] X. Fang and R. O. Claus, "Polarization-independent all-fiber wavelength division multiplexer based on a Sagnac interferometer," Opt. Lett., vol. 20, pp, 21462148, Oct. 1995.

[3] X. Fang, H. Ji, C. T. Allen, K. Demarest, and L. Pelz, "A compound high-order polarization-independent birefringence filter using Sagnac interferometers", IEEE Photon. Technol. Lett., vol. 9, pp. 458-460, Apr. 1997.

[4] Y. Shiquan, L. Zhaohui, D. Xiaoyi, Y. Shuzhong, K. Guiyun, and Z. Qida, "Generation of wavelengthswitched optical pulse from a fiber ring laser with an F-P semiconductor modulator and a HiBi fiber loop mirror", IEEE Photon. Technol. Lett., vol. 14, pp. 774-776, Jun. 2002.

[5] Y. W. Lee, J. Jung, and B. Lee, "Multiwavelengthswitchable SOA-fiber ring laser based on polarization-maintaining fiber loop mirror and polarization beam splitter," IEEE Photon. Technol. Lett., vol. 16, pp. 54-56, Jan. 2004.

[6] R. C. Jones, "New calculus for the treatment of optical systems", J. Opt. Soc. Am., vol. 31, pp. 488-492, Jul. 1941.

[7] F. Heismann and M. S. Whalen, "Broadband resetfree automatic polarization controller", Electron. Lett., vol. 27, pp. 377-379, Feb. 1991.

[8] X. P. Dong, S. Li, K. S. Chiang, M. N. Ng, and B. C. B. Chu, "Multiwavelength erbium-doped fiber laser based on a high-birefringence fiber loop mirror", Electron. Lett., vol. 36, pp. 1609-1610, Sept. 2000.

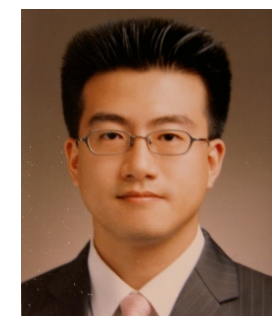

Yong Wook Lee received his B.S., M.S., and Ph.D. degrees in Electrical Engineering in 1998, 2000, and 2004, respectively, from Seoul National University, Seoul, Korea. He worked in Electronics and Telecommunications Research Institute, Daejeon, Korea, from 2004 to 2008. He joined the faculty of the School of Electrical Engineering in Pukyong National University, Busan, Korea, in 2008. His research areas include optical devices for optical sensors and communications such as optical fiber gratings, optical filters, and optical amplifiers as well as optical gating in semiconductor thin films based on photo-induced phase transition. 